\title{
Magnetic Helicity Propagation from Inside the Sun
}

\author{
Dana Longcope \\ Department of Physics, Montana State University, Bozeman, MT 59717
}

\begin{abstract}
Models of twisted flux tube evolution provide a picture of how magnetic helicity is propagated through the solar convection zone into the corona. According to the models, helicity tends toward an approximately uniform lengthdensity along a tube, rather than concentrating at wider portions. Coronal fields lengthen rapidly during active region emergence, requiring additional helicity to propagate from the submerged flux tube. Recent observations of emerging active regions show an evolution consistent with this prediction, and no evidence of helicity concentrating in wider sections.
\end{abstract}

\section{Twisted Flux Tubes}

The solar magnetic field has been modeled as flux tubes, ever since Parker introduced the concept and elucidated many of their properties. His comprehensive monograph, Cosmical Magnetic Fields; Their Origin and Their Activity (Parker 1979), devotes three chapters exclusively to flux tubes. Shortly after its publication, Spruit (1981) introduced a particularly useful non-linear model for the dynamical evolution of thin flux tubes (cross-sectional radius $a \ll H_{p}$ the pressure scale height), by expanding the magnetohydrodynamic equations about the tube's axis. Solutions of these equations for rising active region tubes agree with many observed characteristics of bipolar active regions (see Fisher et al. 2000, and references therein).

Recent interest in the chirality of the solar magnetic field motivated Longcope and Klapper (1997) to extend the standard thin flux tube model to include degrees of freedom they called twist $q(s, t)$ and spin $\omega(s, t)$. These define the azimuthal magnetic field $B_{\phi}=q r \bar{B}$ and plasma velocity $v_{\phi}=\omega r$ small distances $r$ from the axis $\left(\bar{B}=\Phi / \pi a^{2}\right.$ is the mean axial magnetic field in a tube of flux $\Phi)$. The twisted tube carries an axial current $I=2 q \Phi$ along its field lines and an opposite return current along its outer surface.

Expanding both the ideal induction equation and the first moment of the momentum equation (i.e. the equation of axial angular momentum) yields dynamical equations coupling twist and spin (Longcope and Klapper 1997)

$$
\frac{d q}{d t}=\frac{\partial \omega}{\partial s}-q \hat{\mathbf{s}} \cdot \frac{\partial \mathbf{v}_{a}}{\partial s}+\left(\hat{\mathbf{s}} \times \frac{\partial \hat{\mathbf{s}}}{\partial s}\right) \cdot \frac{\partial \mathbf{v}_{a}}{\partial s}, \frac{d \omega}{d t}=v_{\mathrm{A}}^{2} \frac{\partial q}{\partial s}-\omega \frac{d \ln a^{2}}{d t},
$$

where $\mathbf{v}_{a}(s, t)$ is the velocity of the axis at length coordinate $s$, and $\hat{\mathbf{s}}$ is the axis normal. The spin and twist are clearly affected by the dynamics of the tube's axis through $\mathbf{v}_{a}$ and $\dot{a}$ in equations (1). For a slightly twisted tube, one for which $q a \ll 1$, the axis dynamics are not affected by twist or spin. 
In the case of a stationary axis, $\mathbf{v}_{a}=\dot{a}=0$, the spin and twist satisfy a pair of telegrapher's equations

$$
\frac{d q}{d t}=\frac{\partial \omega}{\partial s}, \frac{d \omega}{d t}=v_{\mathrm{A}}^{2} \frac{\partial q}{\partial s},
$$

whose solutions are torsional Alfvén waves. Evidently, to be in equilibrium the tube's twist must be uniformly distributed along its axis, $\partial q / \partial s=0$, regardless of any variation in its radius $a(s)$. This tendency for uniform twist can be viewed as a consequence of either continuity of axial current $I=2 q \Phi$, or a balance of axial torque. A violation, i.e. an interruption of current or a torque imbalance, will lead to a torsional Alfvén pulse, whose propagation will move the variation toward the ends of the tube, thereby "seeking" to restore uniformity.

The tendency for twist uniformity exhibited by the Longcope and Klapper thin twisted flux tube model contradicts a common assertion that twist will concentrate in the widest portions of a flux tube. Such assertions are typically justified by referring to Cosmical Magnetic Fields (Parker 1979), particularly to results from the chapter devoted to the internal structure of axisymmetric flux tubes, not restricted to thin tubes. We return shortly to show that when applied to thin tubes Parker's result actually corroborates the tendency toward twist uniformity, rather than predicting a concentration at wider regions.

\section{Coupling to the Corona}

The thin flux tube model generally fails within the several megameters below the photosphere. Higher still, in the corona, the field is believed to approximate a force-free equilibrium. Torque balance across the intervening layer shows that, except during brief transients, the tube's internal axial current must pass completely into the corona (Longcope and Welsch 2000). The flux tube's surface current, including the axial return current, joins the horizontal surface current of the merging layer. Thus we expect that twist observed in photospheric and coronal magnetic fields, generally of order $|q| \sim 10^{-8} \mathrm{~m}^{-1}$ (Pevstov et al. 1995), reflects a twist in the convection zone flux tubes to which they connect.

Let us assume that the coronal field directly connected to the active region, and thus to the flux tube, consists of closed loops which together fill a finite volume of characteristic scale $\sim d$, the separation between the photospheric poles. In equilibrium these loops will compose a force-free field carrying a net current $I_{c}$ between footpoints. The detailed structure of this field will be complex depending as it does on the distribution of photospheric flux and current. Nevertheless, it will have a relative helicity $H$, which we assume to be finite and, on dimensional grounds, to be $H \simeq C I_{C} \Phi d$, for some constant $C$.

During the process of emergence, the flux tube evidently injects the helicity $H$ into the corona. It must do this while injecting little mass since the ratio of helicity per mass is greater in the corona than in the convection zone by $\sim \rho_{\mathrm{cz}} a^{2} / \rho_{\text {cor }} d^{2} \gg 1$. Thus the emerging flux tube cannot "carry" its helicity into the corona by vertical flow, but must instead "wind" it into to coronal field by horizontal rotational motion. Evidently there is spin, $\omega_{+}$and $\omega_{-}$, in the positive and negative legs of the emerging flux tube which map to photospheric rotations. This spin, which can be decomposed into upward and downward 
propagating torsional Alfvén waves within the sub-photospheric legs, produces a coronal helicity flux (Pevtsov et al. 2003)

$$
d H / d t=2 C \Phi^{2}\left(q_{c} \dot{d}+\dot{q}_{c} d\right)=-\Phi^{2}\left(\omega_{+}+\omega_{-}\right) / 2 \pi,
$$

where $q_{c}=I_{c} / 2 \Phi$ is the value of twist where the thin flux tubes meet the corona.

Pevtsov et al. (2003) used combined MDI and EIT observations of six emerging active regions to test this model of helicity injection. With the exception of one apparently untwisted case, the coronal twist increased from zero to a final value over about 1.5 days. In all five cases with twist, the time history of $d(t)$ and $q_{c}(t)$ were fit by solutions of (2) and (3), with similar values of subphotospheric Alfvén speed and asymptotic twist $q \sim 10^{-8} \mathrm{~m}^{-1}$. According to this interpretation, the separating poles $(\dot{d}>0)$, lengthened the coronal field lines, causing helicity to be drawn from the submerged tubes over a time $d / v_{\mathrm{A}}$.

\section{Internal Twist Distribution}

It is worth comparing this to Parker's model for the internal distribution of twist in an axisymmetric, vertical, equilibrium flux tube. A general axisymmetric field can be written in terms of a flux function ${ }^{1} \psi(r, z)$ as $\mathbf{B}=\nabla \psi \times \nabla \phi+B_{\phi}(r, z) \hat{\boldsymbol{\phi}}$. The flux function rises monotonically from zero on axis to $\psi(a, z)=\Phi / 2 \pi$ at the outer surface, $r=a(z)$.

Since the azimuthal Lorentz force (i.e. magnetic torque) cannot be compensated by pressure, it must vanish in equilibrium, requiring $r B_{\phi}=F(\psi)$ a general function of the flux function. Following Parker we argue that the internal plasma pressure is hydrostatic, and thereby obtain the zero- $\beta$ Grad-Shafranov equation for the flux function

$$
r^{2} \nabla \cdot\left(r^{-2} \nabla \psi\right)=-F^{\prime}(\psi) F(\psi)
$$

The function $F(\psi)$ determines the azimuthal field even where $a(z)$ varies significantly. Parker argues that in any section which can be considered thin, so $\psi \simeq \bar{B} r^{2} / 2, F(\psi)$ can be approximated by the leading term from its series, $F(\psi) \simeq 2 q \psi$. Here $q$ has the same meaning as in the thin-tube dynamical theory of Longcope and Klapper (1997). Since $F(\psi)$ must be the same in every portion of the tube, Parker's theory confirms that $q$ is uniform throughout the equilibrium, regardless of radius.

In a non-thin tube section equation (4) demands a linear force-free field with $\alpha=2 q$. In a thin or approximately straight section $\partial^{2} \psi / \partial s^{2}$ may be dropped yieliding the Lundquist solution found by Parker

$$
\psi=\frac{\Phi}{2 \pi} \frac{r J_{1}(2 q r)}{a J_{1}(2 q a)} \quad, \quad B_{z}=\frac{1}{r} \frac{d \psi}{d r}=\frac{q \Phi}{\pi a J_{1}(2 q a)} J_{0}(2 q r)
$$

where $J_{n}(x)$ is the Bessel function of order $n$. The common assertion about twist concentration follows from the observation that when the tube is wide

\footnotetext{
${ }^{1}$ Parker casts his analysis in terms of a generating function, $f$, related to $\psi$ and $B_{\phi}$.
} 
enough that $2 q a=j_{0,1}=2.405$, the axial field vanishes and the local twist, $B_{\phi} / r B_{z}$, diverges at the tube surface. This large twist is achieved, it is argued, by concentrating all of the tube's twist in this one wider section.

The previous section showed that the dynamically significant quantity is not the local twist, but rather the helicity per unit length,

$$
\mathcal{H}=4 \pi \int_{0}^{a} \psi F(\psi) \frac{d r}{r}=\frac{q \Phi^{2}}{\pi}\left\{\frac{J_{0}^{2}(2 q a)}{J_{1}^{2}(2 q a)}-\frac{J_{0}(2 q a)}{q a J_{1}(2 q a)}+1\right\}
$$

for the Lundquist field (5). In the weakly twisted limit, qa« 1 , the factor in braces approaches one-half, recovering the Longcope and Klapper limit, $\mathcal{H} \simeq$ $q \Phi^{2} / 2 \pi$, independent of tube radius a. Even the pathologically "fat" tube, $2 q a=$ $j_{0,1}$ has helicity only two times greater (in spite of an infinite twist density). Cross-section alone, it seems, can only double the helicity requirement of the tube apex, while its net extension requires far more.

Solar flux tubes have such small values of twist, $q \simeq 10^{-8} \mathrm{~m}^{-1}$, that even their widest sections, while not thin, are still weakly twisted, $q a \ll 1$, and will seek uniform helicity density. Only the coronal field, $d \sim 10^{8} \mathrm{~m}$, might depart from this regime, however, this is also the section most poorly approximated as axisymmetric. The helicity density in a maximum width 1d (Lundquist) field (Parker 1979) is double that of a thin tube, however, 2d axisymmetric fields show slightly different enhancements (Longcope and Welsch 2000).

In conclusion, there is no strong tendency for twist or helicity to move preferentially toward wider portions of flux tubes, or even into the zero- $\beta$ coronal field. It will instead seek uniform density per length, and thus flow to lengthening portions. Observations show evidence of this tendency as emerging active regions fill with helicity through horizontal photospheric motions. This same reasoning predicts that removal of coronal helicity, by cornal mass ejections for instance, should be followed by replenishing photospheric motions driven by the twisted flux tube below. In this way the corona and heliosphere together may function as a sink of helicity produced in the solar interior.

\section{References}

Fisher, G. H., Fan, Y., Longcope, D. W., Linton, M. G., and Abbett, W. P. 2000, Phys. Plasmas 7(5), 2173.

Longcope, D. W. and Klapper, I. 1997, ApJ 488, 443.

Longcope, D. W. and Welsch, B. T. 2000, ApJ 545, 1089.

Parker, E. N. 1979, Cosmical Magnetic Fields, Their Origin and Their Activity, Clarendon Press, Oxford.

Pevtsov, A. A., Canfield, R. C., and Metcalf, T. R. 1995, ApJ 440, L109.

Pevtsov, A. A., Maleev, V., and Longcope, D. W. 2003, ApJ 593, 1217.

Spruit, H. C. 1981, A\&A 98, 155. 